\title{
EXTENDED [C II] EMISSION IN LOCAL LUMINOUS INFRARED GALAXIES
}

\author{
T. Díaz-Santos ${ }^{1}$, L. Armus ${ }^{1}$, V. Charmandaris ${ }^{2,3,4}$, G. StaceY $^{5}$, E. J. Murphy ${ }^{6}$, S. HaAn ${ }^{7}$, S. Stierwalt ${ }^{8}$, S. Malhotra ${ }^{9}$, \\ P. Appleton ${ }^{10}$, H. Inami ${ }^{11}$, G. E. Magdis ${ }^{12}$, D. ElbaZ ${ }^{13}$, A. S. Evans ${ }^{8,14}$, J. M. Mazzarella ${ }^{15}$, J. A. Surace ${ }^{1}$, \\ P. P. van der Werf ${ }^{16}$, C. K. Xu ${ }^{15}$, N. Lu ${ }^{15}$, R. MeIJerinK ${ }^{16,17}$, J. H. Howell ${ }^{15}$, A. O. Petric ${ }^{18,19}$, \\ S. VeIllEuX ${ }^{20,21}$, AND D. B. SANDERS ${ }^{22}$ \\ ${ }^{1}$ Spitzer Science Center, California Institute of Technology, MS 220-6, Pasadena, CA 91125, USA; tanio@ipac.caltech.edu \\ 2 Department of Physics, University of Crete, GR-71003 Heraklion, Greece \\ ${ }^{3}$ Chercheur Associé, Observatoire de Paris, F-75014 Paris, France \\ ${ }^{4}$ Institute for Astronomy, Astrophysics, Space Applications and Remote Sensing, National Observatory of Athens, GR-15236 Athens, Greece \\ ${ }_{5}^{5}$ Department of Astronomy, Cornell University, Ithaca, NY 14853, USA \\ ${ }^{6}$ Observatories of the Carnegie Institution for Science, 813 Santa Barbara Street, Pasadena, CA 91101, USA \\ ${ }^{7}$ CSIRO Astronomy and Space Science, Marsfield, NSW 2122, Australia \\ ${ }^{8}$ Department of Astronomy, University of Virginia, P.O. Box 400325, Charlottesville, VA 22904, USA \\ ${ }^{9}$ School of Earth and Space Exploration, Arizona State University, Tempe, AZ 85287, USA \\ ${ }^{10}$ NASA Herschel Science Center, IPAC, California Institute of Technology, MS 100-22, Cech, Pasadena, CA 91125, USA \\ ${ }^{11}$ National Optical Astronomy Observatory, 950 North Cherry Avenue, Tucson, AZ 85719, USA \\ ${ }^{12}$ Department of Physics, University of Oxford, Keble Road, Oxford OX1 3RH, UK \\ ${ }^{13}$ Laboratoire AIM-Paris-Saclay, CEA/DSM/Irfu, CNRS, Universite Paris Diderot, Saclay, F-91191 Gif-sur-Yvette, France \\ ${ }^{14}$ National Radio Astronomy Observatory, 520 Edgemont Road, Charlottesville, VA 22903, USA \\ ${ }^{15}$ Infrared Processing and Analysis Center, MS 100-22, California Institute of Technology, Pasadena, CA 91125, USA \\ ${ }^{16}$ Leiden Observatory, Leiden University, P.O. Box 9513, NL-2300 RA Leiden, The Netherlands \\ ${ }^{17}$ Kapteyn Astronomical Institute, University of Groningen, P.O. Box 800, NL-9700 AV Groningen, The Netherlands \\ ${ }^{18}$ Gemini Observatory, 670 N. Aohoku Place, Hilo, HI 96720, USA \\ ${ }^{19}$ Astronomy Department, California Institute of Technology, Pasadena, CA 91125, USA \\ ${ }^{20}$ Joint Space-Science Institute, University of Maryland, College Park, MD 20742, USA \\ ${ }^{21}$ Department of Astronomy, University of Maryland, College Park, MD 20742, USA \\ 22 Institute for Astronomy, University of Hawaii, 2680 Woodlawn Drive, Honolulu, HI 96822, USA \\ Received 2014 March 6; accepted 2014 May 12; published 2014 May 27
}

\begin{abstract}
We present Herschel/PACS observations of extended [C II] $157.7 \mu \mathrm{m}$ line emission detected on $\sim 1-10 \mathrm{kpc}$ scales in 60 local luminous infrared galaxies (LIRGs) from the Great Observatories All-sky LIRG Survey. We find that most of the extra-nuclear emission show [C II]/FIR ratios $\geqslant 4 \times 10^{-3}$, larger than the mean ratio seen in the nuclei, and similar to those found in the extended disks of normal star-forming galaxies and the diffuse interstellar medium of our Galaxy. The [C II] "deficits" found in the most luminous local LIRGs are therefore restricted to their nuclei. There is a trend for LIRGs with warmer nuclei to show larger differences between their nuclear and extra-nuclear [C II]/FIR ratios. We find an anti-correlation between [C II]/FIR and the luminosity surface density, $\Sigma_{\mathrm{IR}}$, for the extended emission in the spatially resolved galaxies. However, there is an offset between this trend and that found for the LIRG nuclei. We use this offset to derive a beam filling-factor for the star-forming regions within the LIRG disks of $\sim 6 \%$ relative to their nuclei. We confront the observed trend to photo-dissociation region models and find that the slope of the correlation is much shallower than the model predictions. Finally, we compare the correlation found between $\left[\mathrm{C}\right.$ II]/FIR and $\Sigma_{\mathrm{IR}}$ with measurements of high-redshift starbursting IR-luminous galaxies.
\end{abstract}

Key words: galaxies: ISM - galaxies: nuclei - galaxies: starburst - infrared: galaxies

Online-only material: color figures

\section{INTRODUCTION}

The far-infrared (FIR) [C II] $157.7 \mu \mathrm{m}$ fine-structure emission line is the dominant gas coolant of the neutral interstellar medium (ISM) in normal star-forming galaxies (Malhotra et al. 1997). It mostly arises from photo-dissociation regions (PDR) heated by ultraviolet (UV) radiation emitted by young, massive stars, and can account for up to a few percent of the total IR luminosity of a galaxy (Stacey et al. 1991; Helou et al. 2001). However, the efficiency of the ISM cooling through this emission line is greatly reduced in systems with enhanced radiation field intensity and/or hardness caused by very intense nuclear starbursts (Abel et al. 2009; Stacey et al. 2010; GraciáCarpio et al. 2011; Díaz-Santos et al. 2013). Indeed, compared with normal, quiescently star-forming galaxies, the relative cooling via the $[\mathrm{C} \mathrm{II}]$ line with respect to that carried by dust, as measured by the $[\mathrm{C} \mathrm{II}] / \mathrm{FIR}$ ratio, ${ }^{23}$ decreases by more than an order of magnitude from $\sim 10^{-2}$ in starbursting galaxies with warmer average nuclear dust temperatures and more compact star formation, such as in local luminous infrared galaxies (LIRGs; $L_{\mathrm{IR}} \geqslant 10^{11-12} L_{\odot}$; Díaz-Santos et al. 2013). In addition, in those sources where an active galactic nucleus may contribute to the FIR emission of the galaxy, the [C II]/FIR ratio can drop even further (i.e., there is a larger [C II] "deficit"), up to two orders of magnitude (Díaz-Santos et al. 2013; Farrah et al. 2013).

In our Galaxy and the Magellanic Clouds, [C II] emission arising from star-forming complexes and the diffuse ISM have been studied in great detail (e.g., Wright et al. 1991;

\footnotetext{
${ }^{23}$ FIR is the flux emitted within the $42.5-122.5 \mu \mathrm{m}$ wavelength range as
} originally defined in Helou et al. (1988). 
Shibai et al. 1991; Stacey et al. 1993; Bennett et al. 1994; Poglitsch et al. 1995; Yasuda et al. 2008; Pineda et al. 2012; Lebouteiller et al. 2012). However, before the launch of the Herschel Space Observatory (Pilbratt et al. 2010), there was only a limited number of extragalactic sources for which it was possible to disentangle the [C II] emission arising from spatially resolved star-forming regions from that originating within their nuclei (e.g., Madden et al. 1993; Nikola et al. 2001; Higdon et al. 2003; Kramer et al. 2005; Rodriguez-Fernandez et al. 2006). Now, the Photodetector Array Camera and Spectrometer (PACS; Poglitsch et al. 2010) instrument on board Herschel has enabled us to obtain measurements of the FIR continuum and emission lines on physical scales of a few hundred pc in a significant number of nearby galaxies (Kennicutt et al. 2011; Croxall et al. 2012; Beirão et al. 2012; Kramer et al. 2013), with which we are able to derive the properties $\left(G_{0}, n_{\mathrm{H}}\right)$ of the different phases of the ISM in extragalactic star-forming regions (e.g., Mookerjea et al. 2011; Contursi et al. 2013).

Studying the extra-nuclear [C II] in nearby LIRGs is critical not only for improving our understanding of the physical properties of the ISM in star-forming galaxies, but also for establishing local benchmarks with which to compare measurements of this key emission line in distant IR-luminous galaxies. In fact, observations with the Plateau de Bure Interferometer and ALMA are already starting to find high-redshift galaxies in which [C II] is detected at distances several kpc away from their nuclei (Gallerani et al. 2012; Swinbank et al. 2012; Riechers et al. 2013).

This Letter is a follow-up to our work in Díaz-Santos et al. (2013; hereafter DS13), where we presented the results for our Herschel/PACS [C II] survey regarding the nuclear emission of LIRGs. Here we study the extended [C II] line emission detected for the first time in a large sample of nearby LIRGs. In Section 2 we briefly introduce the sample, observations and analysis of the data. In Section 3 we present our results, and in Section 4 we summarize and discuss them.

\section{SAMPLE AND OBSERVATIONS}

\subsection{The GOALS Sample}

The Great Observatories All-sky LIRG Survey (GOALS; Armus et al. 2009) encompasses the complete luminositylimited sample of the 202 LIRGs and ultraluminous infrared galaxies (ULIRGs) contained in the IRAS Revised Bright Galaxy Sample (Sanders et al. 2003) which, in turn, is a complete fluxlimited sample of 629 galaxies having IRAS $S_{60 \mu m}>5.24 \mathrm{Jy}$ and Galactic latitudes $|b|>5^{\circ}$. There are 180 LIRG and 22 ULIRG systems in GOALS and their median redshift is $z=$ 0.0215 ( or $~ 95.2 \mathrm{Mpc}$ ).

\subsection{Herschel/PACS Observations}

We have obtained Herschel/PACS FIR spectroscopic observations of the [C II] $157.7 \mu \mathrm{m}$ emission line for 200 LIRG systems (241 individual galaxies) in the GOALS sample. Details regarding the processing and analysis of the data are described in DS13. Since the goal of this Letter is to analyze the [C II] deficit in the extra-nuclear regions of LIRGs, in addition to the nuclear [C II] and FIR fluxes calculated in DS13, we have also obtained their spatially integrated fluxes as extracted from a $3 \times 3$ spaxel box $\left(\left(9^{\prime \prime} .4\right)^{2} /\right.$ spaxel $)$ centered around the nuclear spaxel. The extended emission is thus defined as the integrated flux minus the nuclear measurement, after performing a pointsource aperture correction on the nuclear flux to the central $3 \times 3$ spaxel aperture. The same approach was taken to calculate the extended emission of the $63 \mu \mathrm{m}$ dust continuum, which is also used to scale the IRAS FIR flux and $L_{\mathrm{IR}}$ down to the apertures used to extract the PACS spectra (see DS13 for more details). Errors in the line and continuum fluxes have been propagated into the analysis of, e.g., [C II]/FIR. To calculate the IR luminosity surface density $\left(\Sigma_{\mathrm{IR}}\right)$ of the extended emission we use the projected physical area covered by the $3 \times 3$ PACS spaxel box centered around the galaxy minus that of the nuclear spaxel. For the starburst nuclei, however, we use the beam-corrected MIR sizes estimated from the Spitzer/IRS spectra and MIPS imaging, $\Sigma_{\mathrm{IR}}=L_{\mathrm{IR}} / \pi r_{\mathrm{MIR}}^{2}$ (see DS13).

A galaxy is considered to be spatially resolved when more than $30 \%$ of the [C II] and FIR continuum flux is extended, as defined above. This threshold is robust against observations of unresolved sources in which mis-pointings of up to $\sim 3^{\prime \prime}$ would introduce a systematic underestimation of the nuclear flux that would mimic extended emission. ${ }^{24}$ Because of possible contamination from a nearby companion galaxy, we exclude from this study LIRG systems in which individual galaxies are closer than 23.5 from each other (one-half of the PACS field of view). A total of 60 galaxies ( $25 \%$ of the sample) show extended $[\mathrm{C}$ II $]$ and FIR emission meeting these constraints. Their average extended [C II] emission is $52 \%$, with a maximum of $75 \%$. The projected radial distance at which the extended emission is detected ranges from 1 to $\sim 12.5 \mathrm{kpc}$ for the closest and farthest resolved galaxy, respectively, with a median of $4.3 \mathrm{kpc}$. While more luminous GOALS systems tend to be located farther away, a K-S test comparing the distance and luminosity distributions of the GOALS galaxies with $L_{\mathrm{IR}} \leqslant 10^{12} L_{\odot}$ and those of galaxies classified as extended in this work, confirms that both subsamples are indistinguishable in both quantities ( $D$-values of 0.20 and 0.15 and $p$-values of 0.04 and 0.22 , respectively).

\section{RESULTS}

\section{1. [C II] Deficit and FIR Colors of Extra-nuclear Star Formation}

Figure 1 shows the $\left[\mathrm{C}\right.$ II]/FIR ratio as a function of the $S_{v}$ $63 \mu \mathrm{m} / S_{v} 158 \mu \mathrm{m}$ continuum flux density ratio $(63 / 158 \mu \mathrm{m}$ hereafter $)^{25}$ for the extra-nuclear emission observed in spatially resolved LIRGs in the GOALS sample. As expected, the extended emission regions follow the trend found for the LIRG nuclei (DS13) suggesting that the [C II] deficit is related to the average temperature of the dust $\left(T_{\text {dust }}\right)$. However, the extended regions show smaller [C $\mathrm{II}]$ deficits (stronger [C $\mathrm{II}]$ to FIR emission) and lower $63 / 158 \mu \mathrm{m}$ ratios than the majority of the nuclei. The extended emission has a mean and median $\left[\mathrm{C}_{\mathrm{II}}\right] /$ FIR ratio of $(6.9 \pm 2.6) \times 10^{-3}$ and $6.6 \times 10^{-3}$, respectively, which is a factor 2 larger than the LIRG nuclei. Figure 1 also reveals that the fractional extent of the [C II] emission does not correlate with the FIR color of the extra-nuclear regions suggesting that galaxies with larger extended emission fractions do not have systematically colder or warmer $T_{\text {dust }}$. In the majority of LIRGs the extended emission has $63 / 158 \mu \mathrm{m} \lesssim 1$ and

\footnotetext{
24 We have used the new task

http://herschel.esac.esa.int/hcss-doc-12.0/load/pacs_urm/html/herschel.pacs. spg.spec.SpecExtendedToPointCorrectionTask.html

"specExtendedToPointCorrection" available in HIPEv12 to estimate that a 3 " mis-pointing within the central spaxel would artificially increase the fraction of extended emission only up to $23 \%$.

25 For comparisons with IRAS-derived FIR colors, an equation to convert PACS $63 / 158 \mu \mathrm{m}$ into IRAS $60 / 100 \mu \mathrm{m}$ can be found in the Appendix of DS13.
} 


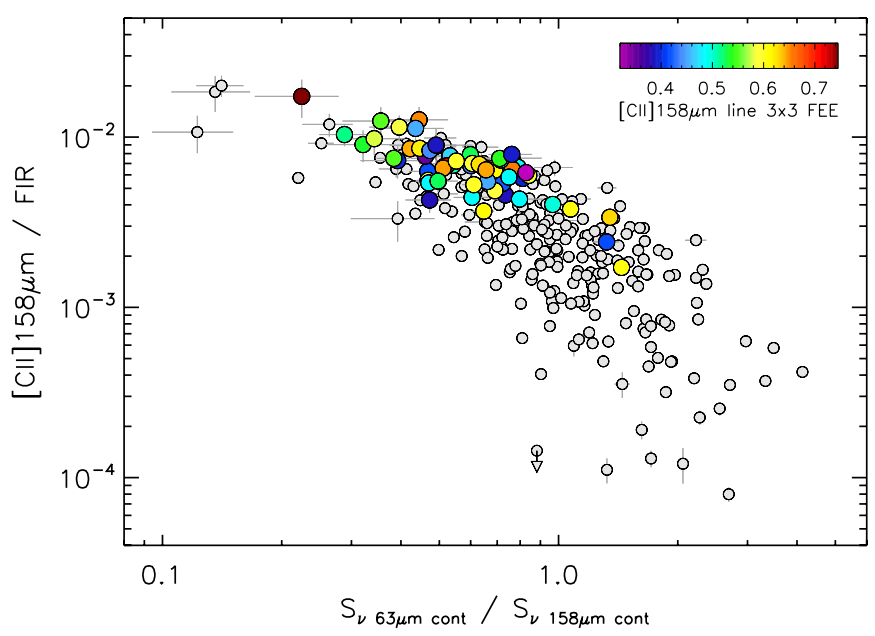

Figure 1. Ratio of [C II] $157.7 \mu \mathrm{m}$ to FIR flux as a function of the $S_{v} 63 \mu \mathrm{m} /$ $S_{v} 158 \mu \mathrm{m}$ continuum flux density ratio for the extended (colored circles) and nuclear regions (gray circles; taken from DS13) of spatially resolved galaxies in the GOALS sample. Circles of different colors (see color bar) indicate the fraction of $[\mathrm{C} \mathrm{II}]$ emission that is extended (FEE) outside of the aperturecorrected central PACS spaxel containing the nuclear emission (see Section 2.2).

(A color version of this figure is available in the online journal.)

$[\mathrm{C}$ II $] /$ FIR $\geqslant 4 \times 10^{-3}$, similar to those found in the extended disks of normal star-forming galaxies (Madden et al. 1993; Nikola et al. 2001; Mookerjea et al. 2011; Croxall et al. 2012), as well as in the diffuse component of the ISM in the Galactic plane (Shibai et al. 1991; Bennett et al. 1994; Nakagawa et al. 1998; Yasuda et al. 2008). This is consistent with our previous findings based on Spitzer/IRS spectroscopy showing that the physical properties of the extra-nuclear, kpc-scale star formation in LIRGs are very similar to those of normal star-forming galaxies with lower IR luminosities $\left(L_{\mathrm{IR}} \sim 10^{10-11} L_{\odot}\right)$, and that the diversity of their integrated MIR spectra is driven by the nuclear, few central kpc starburst (Díaz-Santos et al. 2011).

The differences between the nuclear and extended emission are more clearly seen in Figure 2, which shows that the ratio between the FIR color of the extra-nuclear and nuclear regions is clearly correlated with the excess of [C II] deficit seen in the nuclei with respect to that observed in the disks, regardless of the distance at which the extended emission is measured or on the distance to the galaxy (see color-coding). That is, for a given $T_{\text {dust }}$ of the extended emission, LIRGs with warmer nuclei show larger differences between their nuclear and extra-nuclear [C II] deficits.

\subsection{Extended IR Luminosity Surface Densities}

As shown in DS13, there is a clear trend for pure starforming LIRGs having more compact IR emission to display lower [C II]/FIR ratios. Figure 3(a) shows that the extended emission regions in spatially resolved galaxies follow an anticorrelation between $\left[\mathrm{C}\right.$ II] $/ F I R$ and $\Sigma_{\mathrm{IR}}$ similar to that seen for the LIRG nuclei, with slopes of $-0.39 \pm 0.07$ and $-0.35 \pm 0.03$ respectively, suggesting that these correlations are likely driven by the same physical process. However, the extra-nuclear regions (green filled circles) are offset by more than an order of magnitude toward lower $\Sigma_{\mathrm{IR}}$ and show higher [C II]/FIR ratios on average than their nuclei (red open circles).

To understand this offset we can decompose it as a simultaneous shift in [C II]/FIR and $\Sigma_{\text {IR }}$ along the slope of the observed trend, plus a horizontal shift in $\Sigma_{\mathrm{IR}}$. The shift along the correlation is determined by the ratio between the median

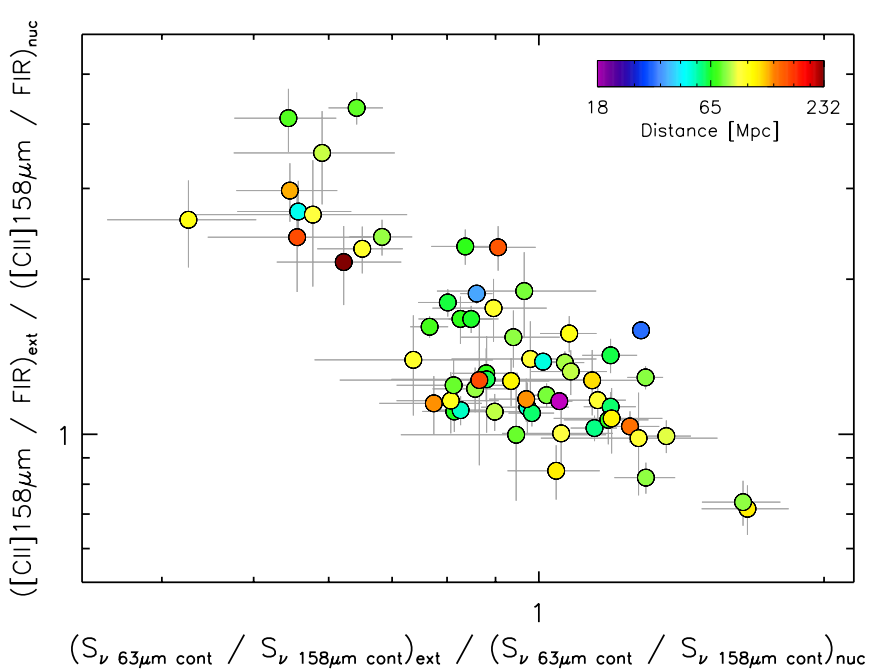

Figure 2. Ratio of $[\mathrm{CII}] /$ FIR between the extended and nuclear emission of LIRGs as a function of the extended to nuclear FIR color. Circles of different colors indicate the distance to the galaxy.

(A color version of this figure is available in the online journal.)

[C II]/FIR measured in the extra-nuclear regions and that of the starburst nuclei. The additional horizontal shift in $\Sigma_{\mathrm{IR}}$ represents the beam filling-factor of the star-forming regions in the LIRG disks with respect to that of the nuclei. We estimate this relative filling-factor to be $0.06_{-0.04}^{+0.13}$ by minimizing the difference between the median $\Sigma_{\text {IR }}$ of the extended and nuclear emissions (see Figure 3(b)). The uncertainties in the filling factor account for the dispersion of the [C II]/FIR versus $\Sigma_{\text {IR }}$ correlation in the $\mathrm{x}$-axis as well as for variations in the properties of the ISM that would modify the [C II]/FIR ratio, such as changes in the fractional contribution of [C II] emission associated with the diffuse ionized medium.

This methodology has been recently used in combination with the PDR models developed by Wolfire et al. (1990) to argue for the presence of large-scale star formation in highredshift galaxies (Hailey-Dunsheath et al. 2010; Stacey et al. 2010; Ferkinhoff et al. 2014). These models relate the radius $(R)$ and $L_{\mathrm{IR}}$ of the starburst region to the strength of the UV interstellar radiation field (ISRF), $G_{0}$, in units of the local Galactic value $\left(1.6 \times 10^{-3} \mathrm{erg} \mathrm{s}^{-1} \mathrm{~cm}^{-2}\right.$; Habing 1968). We can compare the relative beam filling factor of the extranuclear star-forming regions derived from Figure 3 to that predicted by the models. Wolfire et al. (1990) consider two geometric configurations for the starburst region. One in which the molecular clouds are immerse in a smooth ISRF and another in which a central, ionizing source is surrounded by a geometrically thick, molecular medium. Each one follows a relation such that $G_{0} \propto \lambda L_{\mathrm{IR}} / R^{3}$ and $G_{0} \propto L_{\mathrm{IR}} / R^{2}$, respectively, with $\lambda$ being the mean free path of a UV photon within the starburst region. If we assume a hydrogen density, $n_{\mathrm{H}}$, in the range of $\sim 10^{2-5} \mathrm{~cm}^{-3}$, we can use Figure 4 of Stacey et al. (2010), which is based on the PDR models from Kaufman et al. (1999), to estimate the [C II]/FIR ratio as a function of $G_{0}$. Although neither model fits our observed trend of [C II]/FIR with $\Sigma_{\mathrm{IR}}$, the model with a compact, nearby ionizing source, i.e., $G_{0} \propto R^{-2}$ (dashed line in Figure 3), is closest to our data. If we use M82 as our reference starburst (with the following properties: $G_{0}=1000$ and $L_{\mathrm{IR}} \sim 3 \times 10^{10} L_{\odot}$, Lord et al. 1996; $D \sim 420$ pc, Joy et al. 1987), the beam filling factor predicted by this configuration for the extra-nuclear star-forming 

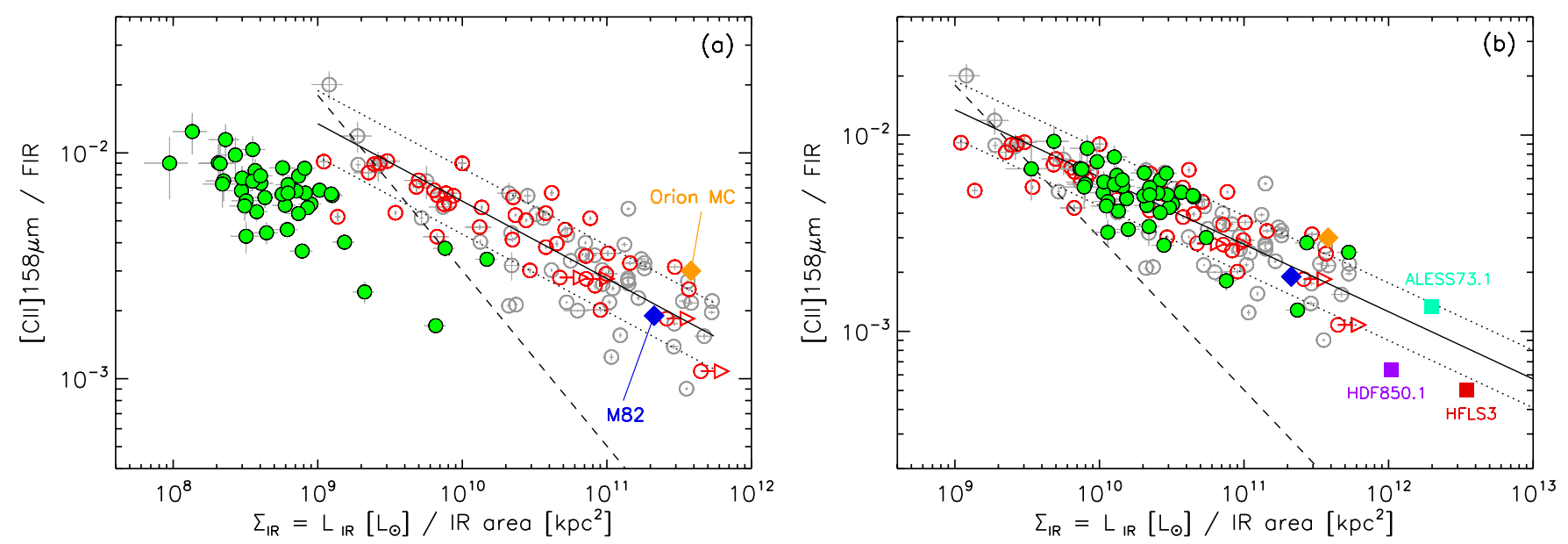

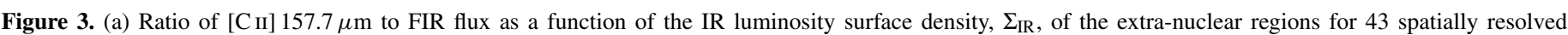

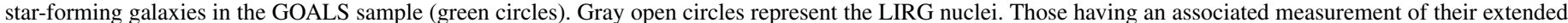

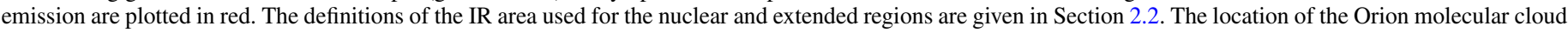

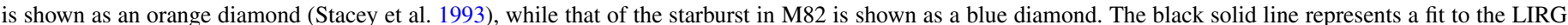

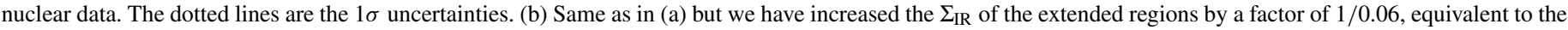

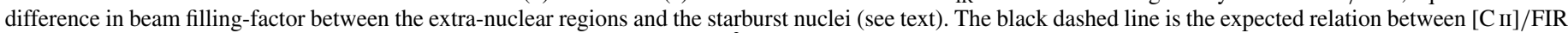

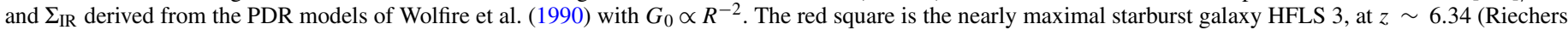

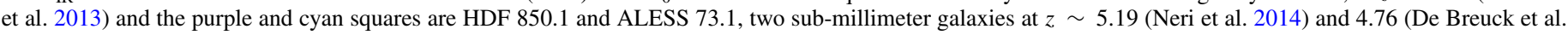
2014).

(A color version of this figure is available in the online journal.)

regions would be $\sim 1 \%$ that of M82. This value is significantly lower than our inferred $6 \%$, when we compare them to the LIRG nuclei using the observed correlation in Figure 3. We explore the reasons for this difference below.

\section{DISCUSSION}

The discrepancy between the filling factor derived from our data and that estimated using the PDR models is due to the slope of the trend found for the LIRG nuclei, which is still significantly shallower than that implied by the PDR model. Using the relationship between [C II]/FIR and $G_{0}$ in (Stacey et al. 2010), ${ }^{26}$ a spatial distribution of the star formation of $G_{0} \propto L_{\mathrm{IR}} / R^{2}$ is equivalent to $\log ([\mathrm{C}$ II $] / F I R) \propto-0.78 \times \log \left(L_{\mathrm{IR}} / R^{2}\right)$ (see dashed line in Figure 3 ). This significantly differs from the slope fitted to the data, -0.35 . The difference in the slopes may arise at least in part from the fact that as the radiation field becomes more intense, not only the gas heating efficiency is reduced due to the increased ionization of small dust particles but dust grains could also be intercepting a larger fraction of the UV radiation before it reaches the PDR (see Abel et al. 2009). Since this dust will be warmer, the natural consequence is also a smaller size for the starburst, as measured in the MIR, and hence a larger $\Sigma_{\mathrm{IR}}$. In fact, the $[\mathrm{C}$ II]/FIR ratio is correlated with the depth of the $9.7 \mu \mathrm{m}$ silicate absorption feature (DS13), which measures the obscuration toward the warm dust continuum originating from the nuclear star-forming regions. It is therefore a combination of a decreased heating efficiency plus warm dust inside the PDR and likely within the $\mathrm{H}$ II regions, that causes a drop in

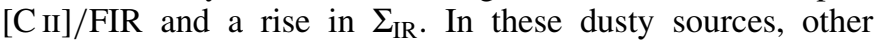
FIR emission lines arising from the dense ionized gas (e.g., [N II], [N III], or [O III]) also show deficits with respect to the FIR emission (Graciá-Carpio et al. 2011), suggesting that a

26 See also the Photo Dissociation Region Toolbox (PDRT) models: http://dustem.astro.umd.edu/pdrt/ (Tielens \& Hollenbach 1985; Wolfire et al. 1990; Hollenbach et al. 1991; Kaufman et al. 1999). significant amount of dust may indeed be present inside the $\mathrm{H}$ II regions.

The PDR model presented in Figure 3 not only has a steeper slope than the trends observed for the nuclei and extended regions of local LIRG, but the majority of the nuclear data, mostly above $\Sigma_{\mathrm{IR}} \sim 10^{10} L_{\odot} \mathrm{kpc}^{-2}$, are located to the right of the model prediction. Since the model assumes a constant filling-factor of unity for the starburst region, this implies a beam filling-factor for the LIRG nuclei increasingly larger than unity. A possible interpretation is that, at a given [C II]/FIR ratio, we can consider the trend with $\Sigma_{\text {IR }}$ found for the LIRG nuclei as "maximal," i.e., where the nuclear starbursts have the highest - most compact- $\Sigma_{\mathrm{IR}}$ possible, with multiple starforming regions overlapping along the line of sight within the beam.

These findings may be useful for studies of IR-luminous galaxies detected in high-redshift surveys for which it is not possible to resolve the [C II] or IR emission. As an example, in Figure 3(b) we show HFLS 3 (Riechers et al. 2013), a nearmaximal starburst galaxy at $z \sim 6.34$, and two sub-millimeter galaxies, HDF 850.1 (Neri et al. 2014) and ALESS 73.1 (De Breuck et al. 2014), at $z \sim 5.19$ and 4.76, respectively. All galaxies have a [C II] deficit and $\Sigma_{\mathrm{IR}}$ broadly consistent with the extrapolation of the correlation derived from the nuclei of local LIRGs (DS13). We note that the $\Sigma_{\text {IR }}$ of the high- $z$ sources may actually be underestimated because their sizes are measured in the rest-frame FIR continuum, which is dominated by the cold dust. But if the reported sizes are correct, the offset of HFLS 3 and HDF 850.1 may imply beam filling factors for the star-forming complexes 2-5 times larger than local LIRG disks would have with similar [C II]/FIR-or, alternatively, these high- $z$ sources would be showing properties similar to those of local ULIRG nuclei in GOALS, but extended over larger physical scales.

We thank the anonymous referee for her/his useful comments and suggestions. We would like to thank Mark Wolfire 
for providing us with very useful insights regarding the PDR models. V.C. would like to acknowledge partial support from the EU F97 grant PIRSES-GA-2012-316788. This work is based on observations made with the Herschel Space Observatory, a European Space Agency Cornerstone Mission with science instruments provided by European-led Principal Investigator consortia and significant participation from NASA. The Spitzer Space Telescope is operated by the Jet Propulsion Laboratory, California Institute of Technology, under NASA contract 1407. This research has made use of the NASA/IPAC Extragalactic Database (NED), which is operated by the Jet Propulsion Laboratory, California Institute of Technology, under contract with the National Aeronautics and Space Administration, and of NASA's Astrophysics Data System (ADS) abstract service.

\section{REFERENCES}

Abel, N. P., Dudley, C., Fischer, J., Satyapal, S., \& van Hoof, P. A. M. 2009, ApJ, 701,1147

Armus, L., Mazzarella, J. M., Evans, A. S., et al. 2009, PASP, 121, 559

Beirão, P., Armus, L., Helou, G., et al. 2012, ApJ, 751, 144

Bennett, C. L., Fixsen, D. J., Hinshaw, G., et al. 1994, ApJ, 434, 587

Contursi, A., Poglitsch, A., Grácia Carpio, J., et al. 2013, A\&A, 549, A118

Croxall, K. V., Smith, J. D., Wolfire, M. G., et al. 2012, ApJ, 747, 81

De Breuck, C., Williams, R. J., Swinbank, M., et al. 2014, arXiv:1404.2295

Díaz-Santos, T., Armus, L., Charmandaris, V., et al. 2013, ApJ, 774, 68

Díaz-Santos, T., Charmandaris, V., Armus, L., et al. 2011, ApJ, 741, 32

Farrah, D., Lebouteiller, V., Spoon, H. W. W., et al. 2013, ApJ, 776, 38

Ferkinhoff, C., Brisbin, D., Parshley, S., et al. 2014, ApJ, 780, 142

Gallerani, S., Neri, R., Maiolino, R., et al. 2012, A\&A, 543, A114

Graciá-Carpio, J., Sturm, E., Hailey-Dunsheath, S., et al. 2011, ApJL, 728, L7

Habing, H. J. 1968, BAN, 19, 421

Hailey-Dunsheath, S., Nikola, T., Stacey, G. J., et al. 2010, ApJL, 714, L162

Helou, G., Khan, I. R., Malek, L., \& Boehmer, L. 1988, ApJS, 68, 151
Helou, G., Malhotra, S., Hollenbach, D. J., Dale, D. A., \& Contursi, A 2001, ApJL, 548, L73

Higdon, S. J. U., Higdon, J. L., van der Hulst, J. M., \& Stacey, G. J. 2003, ApJ, 592,161

Hollenbach, D. J., Takahashi, T., \& Tielens, A. G. G. M. 1991, ApJ, 377,192

Joy, M., Lester, D. F., \& Harvey, P. M. 1987, ApJ, 319, 314

Kaufman, M. J., Wolfire, M. G., Hollenbach, D. J., \& Luhman, M. L. 1999, ApJ, 527,795

Kennicutt, R. C., Calzetti, D., Aniano, G., et al. 2011, PASP, 123, 1347

Kramer, C., Abreu-Vicente, J., García-Burillo, S., et al. 2013, A\&A, 553, A114

Kramer, C., Mookerjea, B., Bayet, E., et al. 2005, A\&A, 441, 961

Lebouteiller, V., Cormier, D., Madden, S. C., et al. 2012, A\&A, 548, A91

Lord, S. D., Malhotra, S., Lim, T., et al. 1996, A\&A, 315, L117

Madden, S. C., Geis, N., Genzel, R., et al. 1993, ApJ, 407, 579

Malhotra, S., Helou, G., Stacey, G., et al. 1997, ApJL, 491, L27

Mookerjea, B., Kramer, C., Buchbender, C., et al. 2011, A\&A, 532, A152

Nakagawa, T., Yui, Y. Y., Doi, Y., et al. 1998, ApJS, 115, 259

Neri, R., Downes, D., Cox, P., \& Walter, F. 2014, arXiv:1401.2396

Nikola, T., Geis, N., Herrmann, F., et al. 2001, ApJ, 561, 203

Pilbratt, G. L., Riedinger, J. R., Passvogel, T., et al. 2010, A\&A, 518, L1

Pineda, J. L., Mizuno, N., Röllig, M., et al. 2012, A\&A, 544, A84

Poglitsch, A., Krabbe, A., Madden, S. C., et al. 1995, ApJ, 454, 293

Poglitsch, A., Waelkens, C., Geis, N., et al. 2010, A\&A, 518, L2

Riechers, D. A., Bradford, C. M., Clements, D. L., et al. 2013, Natur, 496, 329

Rodriguez-Fernandez, N. J., Braine, J., Brouillet, N., \& Combes, F. 2006, A\&A, 453,77

Sanders, D. B., Mazzarella, J. M., Kim, D.-C., Surace, J. A., \& Soifer, B. T. 2003, AJ, 126, 1607

Shibai, H., Okuda, H., Nakagawa, T., et al. 1991, ApJ, 374, 522

Stacey, G. J., Geis, N., Genzel, R., et al. 1991, ApJ, 373, 423

Stacey, G. J., Hailey-Dunsheath, S., Ferkinhoff, C., et al. 2010, ApJ, 724, 957

Stacey, G. J., Jaffe, D. T., Geis, N., et al. 1993, ApJ, 404, 219

Swinbank, A. M., Karim, A., Smail, I., et al. 2012, MNRAS, 427, 1066

Tielens, A. G. G. M., \& Hollenbach, D. 1985, ApJ, 291, 722

Wolfire, M. G., Tielens, A. G. G. M., \& Hollenbach, D. 1990, ApJ, 358, 116

Wright, E. L., Mather, J. C., Bennett, C. L., et al. 1991, ApJ, 381, 200

Yasuda, A., Nakagawa, T., Spaans, M., Okada, Y., \& Kaneda, H. 2008, A\&A, 480,157 